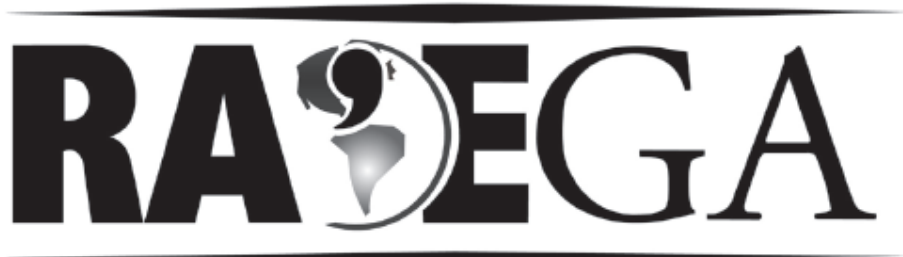

O ESPAÇO GEOGRÁFICO EM ANÁLISE

\title{
CLASSIFICAÇÃO DE DETRITOS LENHOSOS ACUMULADOS EM TRECHOS DO RIO GUABIROBA, GUARAPUAVA (PR)
}

\section{WOODY DEBRIS CLASSIFICATION IN GUABIROBA RIVER, GUARAPUAVA, BRAZIL}

\section{Oscar Vicente Quinonez Fernandez ${ }^{1}$}

\author{
Andrey Luis Binda ${ }^{2}$
}

\section{RESUMO}

O presente trabalho reporta a aplicação da classificação de detritos lenhosos proposta por Montgomery (2008) em trechos do rio Guabiroba, Guarapuava (PR). O termo detrito lenhoso é aplicado a todo material florístico oriundo da mata ciliar e cumpre um importante papel na dinâmica dos processos fluviais. O sistema classificatório consiste em uma nomenclatura alfanumérica (exemplo: B2, A3) que representa o comprimento (código alfabético) e o diâmetro (código numérico) dos resíduos arbóreos. Esta classificação constitui um excelente sistema para inventariar as dimensões físicas dos troncos e galhos acumulados no leito fluvial e sua aplicação no rio Guabiroba serve como um exemplo para descrever quantitativamente a população dos resíduos lenhosos amontoados nas seções estudadas.

Palavras-chave: geomorfologia fluvial; detritos lenhosos; classificação fluvial.

\section{ABSTRACT}

This paper reports the application of classification of woody debris proposed by Montgomery (2008) in reaches of the Guabiroba stream, Guarapuava, Parana State, Brazil. The term wood debris is applied to all organic materials derived

\footnotetext{
${ }^{1}$ Geólogo, doutor em Geociências, professor do Curso de Geografia (Marechal C. Rondon)- Universidade Estadual do Oeste do Paraná (UNIOESTE). E-mail: oscar.fernandez@unioeste.br

${ }^{2}$ Geógrafo, mestre em Geografia, professor do curso de Geografia - Universidade Federal da Fronteira Sul (UFFS). E-mail: abinda@uffs.edu.br
} 
from riparian vegetation plays an important role in the dynamics of the fluvial processes. The classificatory system consisting of an alphanumeric nomenclature (example: B2, A3) which represents the length (code letter) and diameter (numerical code) of wood debris. This classification is an excellent system for inventorying the physical dimensions of the trunks and branches accumulated in the riverbed and its application in Guabiroba stream serves as an example to describe quantitatively the population or woody debris piled in the sections studied.

Key words: fluvial geomorphology; woody debris; classification system.

\section{INTRODUÇÃO}

O termo "detrito lenhoso" (woody debris, organic debris) é aplicado a todo material florístico (troncos, galhos, raízes e folhas ) acumulados no leito fluvial e planície de inundação (LINSTEAD e GURNELL, 1999; GURNELL et al., 2002; WEBB e ERSKINE, 2003; WALLERSTEIN e THORNE, 2004). Os detritos lenhosos podem ser subdivididos em: (1) pequenos detritos orgânicos (fine organic debris) e são formados por folhas e pequenos galhos; (2) pequenos detritos lenhosos (fine woody debris) constituídos por material lenhoso cujo comprimento seja superior a $0,5 \mathrm{~m}$ e diâmetro variando entre 0,03 e $0,1 \mathrm{~m} \mathrm{e}$, (3) grandes detritos lenhosos (large woody debris) quando os detritos apresentam comprimento superior a $0,5 \mathrm{~m}$ e diâmetro maior do que 0,1 m (GOMl et al., 2003; LESTER et al., 2006).

Os entulhos arbóreos desempenham um importante papel modificador sobre os processos fluviais, que por sua vez alteram os aspectos morfológicos e sedimentológicos dos cursos de água (KELLER e SWANSON, 1979; BROOKS et al., 2003; DANIELS e RHOADS, 2003; HASCHENBURGER e RICE, 2004; DANIELS, 2006). Para Bevan (1948; 1949 apud KELLER e SWANSON, 1979), os grandes detritos lenhosos podem ser considerados os maiores agentes modificadores da morfologia em canais florestados. Já os pequenos detritos lenhosos assumem um papel secundário na modificação das características morfológico-hidráulicas dos cursos de água (FARIA, 2000; LENZI et al., 2006).

A presença dos troncos e galhos também é importante no aumento da diversidade ecológica dos habitats aquáticos (GIPPEL et al.,1994; GURNELL et al., 1995, 2002; SUNDBAUM e NAESLUND, 1998; BRAGG et al., 2000; Mac 
NALLY et al., 2001; KEIM et al., 2002; MONTGOMERY et al., 2003; MONTGOMERY e PIEGAY, 2003; LESTER et al., 2006).

As acumulações de detritos lenhosos podem ser descritas utilizando inúmeras variáveis referentes aos troncos ou às acumulações, tais como: comprimento, diâmetro, orientação com referencia ao fluxo, grau de fixação, função de cada resíduo nas acumulações, espécie vegetal ao qual pertence cada tronco, idade, etc. (WOHL et al., 2010). Recentemente, Montgomery (2008) publicou uma proposta de classificação dos entulhos lenhosos, baseada no comprimento e diâmetro dos troncos e galhos. No presente trabalho é relatada a aplicação da classificação de Montgomery (2008) no rio Guabiroba, Guarapuava (PR) e o papel dos detritos lenhosos nos processos de erosão e deposição no leito fluvial.

\section{CLASSIFICAÇÃO DE DETRITOS LENHOSOS}

Numa acumulação de detritos lenhosos (Figura 1) as peças podem ser identificadas baseadas na sua integridade e função estrutural em três tipos grupos: (1) membros-chave (key-members): correspondem àquelas peças estáveis (troncos maiores), que iniciaram a formação da acumulação retendo outras peças menores; (2) membros-suportados (racked members): são aqueles resíduos lenhosos que se encontram fixados pelos membros-chave e, (3) membros soltos (loose members): compreendem pequenos detritos lenhosos e/ou orgânicos que preenchem os espaços intersticiais da acumulação (ABBE e MONTGOMERY, 2003). 


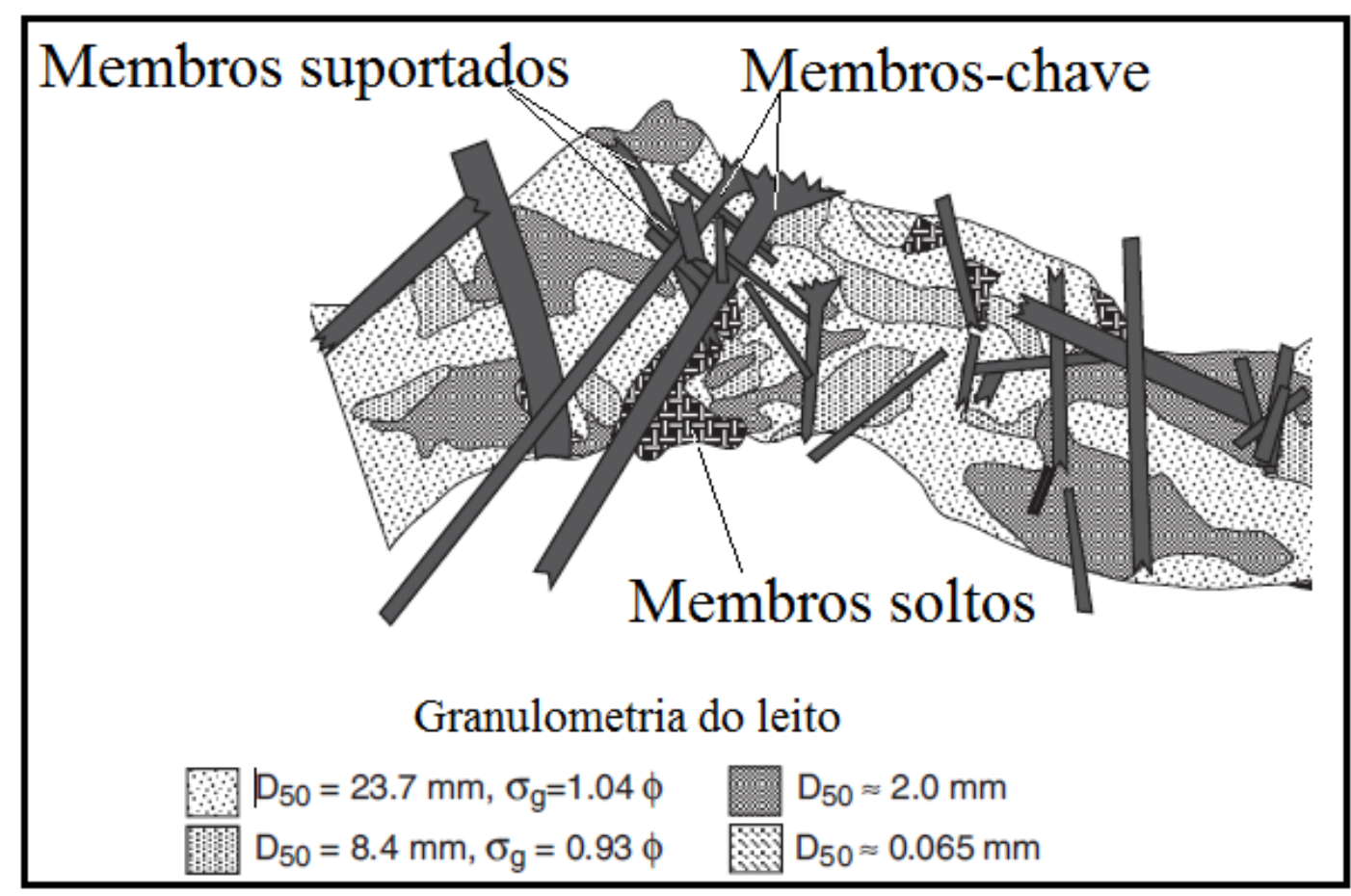

Figura 1: Acumulação dos detritos lenhosos com representação esquemática dos membros-chave, suportado e soltos (Modificada de MONTGOMERY et al., 2003).

A formação de uma acumulação de detritos lenhosos necessita, inicialmente, de um ou mais membros-chave que se encontrem estáveis no leito e promovam o acumulo de peças firmemente ancoradas (membro suportado) nos membros-chave. Os detritos suportados, por sua vez, promovem a retenção de detritos soltos e partículas de matéria orgânica. Assim, o conjunto de membros-chave estável inicia, não somente uma acumulação de resíduos arbóreos, mas também desencadeia uma sequência de mudanças que afetam as características físicas e biológicas do rio (BRAUDICK e GRANT, 2001; ABBE e MONTGOMERY, 2003; YOUNG et al., 2006).

Montgomery (2008) propôs uma classificação para agrupar os detritos lenhosos com base no seu comprimento e diâmetro, empregando uma escala geométrica, que utiliza potências de 2 e tendo $0 \mathrm{~m}$ como ponto de partida. Esta classificação é constituída por uma nomenclatura alfanumérica composta por códigos alfabéticos e numéricos (ex. B2, C3, et.). O primeiro classifica o detrito lenhoso quanto ao seu comprimento e o segundo se refere ao seu diâmetro 
(Tabela 1). Assim, por exemplo, um tronco com $2,5 \mathrm{~m}$ de comprimento e 0,45 $\mathrm{m}$ de diâmetro será classificado como do tipo $\mathrm{C} 4$ e outro com $6,0 \mathrm{~m}$ de comprimento e 0,15 m de diâmetro será agrupado no tipo D2.

Tabela 1: Sistema de classificação dos detritos lenhosos com base no comprimento e diâmetro (MONTGOMERY, 2008).

\begin{tabular}{|c|c|c|c|}
\hline $\begin{array}{c}\text { Códigos } \\
\text { alfabéticos }\end{array}$ & $\begin{array}{c}\text { Comprimento dos } \\
\text { detritos lenhosos }(\mathrm{m})\end{array}$ & $\begin{array}{c}\text { Códigos } \\
\text { numéricos }\end{array}$ & $\begin{array}{c}\text { Diâmetro dos detritos } \\
\text { lenhosos }(\mathrm{m})\end{array}$ \\
\hline A & $0 \mathrm{a} 1$ & 1 & $0 \mathrm{a} 0,1$ \\
\hline $\mathrm{B}$ & $1 \mathrm{a} 2$ & 2 & $0,1 \mathrm{a} 0,2$ \\
\hline C & $2 \mathrm{a} 4$ & 3 & $0,2 \mathrm{a} 0,4$ \\
\hline $\mathrm{D}$ & $4 \mathrm{a} 8$ & 5 & $0,4 \mathrm{a} 0,8$ \\
\hline E & $8 \mathrm{a} 16$ & 6 & $0,8 \mathrm{a} 1,6$ \\
\hline F & $16 \mathrm{a} 32$ & 7 & $1,6 \mathrm{a} 3,2$ \\
\hline $\mathrm{G}$ & $>32$ & & $>3,2$ \\
\hline
\end{tabular}

A escala geométrica foi adotada inicialmente pelo especialista em sedimentogia norte-americano J. A. Udden em 1898 e modificada por C. K. Wentworth em 1922, sendo conhecida a partir de então como escala de Udden-Wentworth (PETTIJOHN et al., 1973).

A proposta de Montgomery (2008) segue a classificação de Rosgen (1994) que também emprega uma nomenclatura alfanumérica para agrupar cursos de água com base nos aspectos morfológicos do canal (códigos alfabéticos) e características granulométricas do material de fundo (códigos numéricos). Na classificação de Rosgen (1994) a diferenciação do tipo de material de fundo obedece a escala de Udden-Wentworth e atribui o valor de 1 para leito rochoso, 2 para leito composto por matacões $(>256 \mathrm{~mm}), 3$ para materiais formados por blocos (64-256 mm), 4 para leitos capeados por seixos e finalmente 5 e 6 para leitos formados por areia e silte+argila, respectivamente. Assim por exemplo, na classificação de Rosgen, um canal com padrão meândrico e leito lamoso é classificado como do tipo E6.

\section{DESCRIÇÃO DA ÁREA DE ESTUDO}

A bacia hidrográfica do Rio Guabiroba está localizada no município de Guarapuava, mesorregião centro-sul do Estado do Paraná (Figura 2). A área da bacia é de $23,9 \mathrm{~km}^{2}$ e o canal principal apresenta $10,4 \mathrm{~km}$ de extensão, 
desde sua nascente até a foz no Rio das Pedras (Sistema do Rio JordãoIguaçu). O rio Guabiroba é caracterizado por apresentar leito misto, com trechos rochosos intercalados por segmentos aluviais (BINDA e LIMA, 2008).

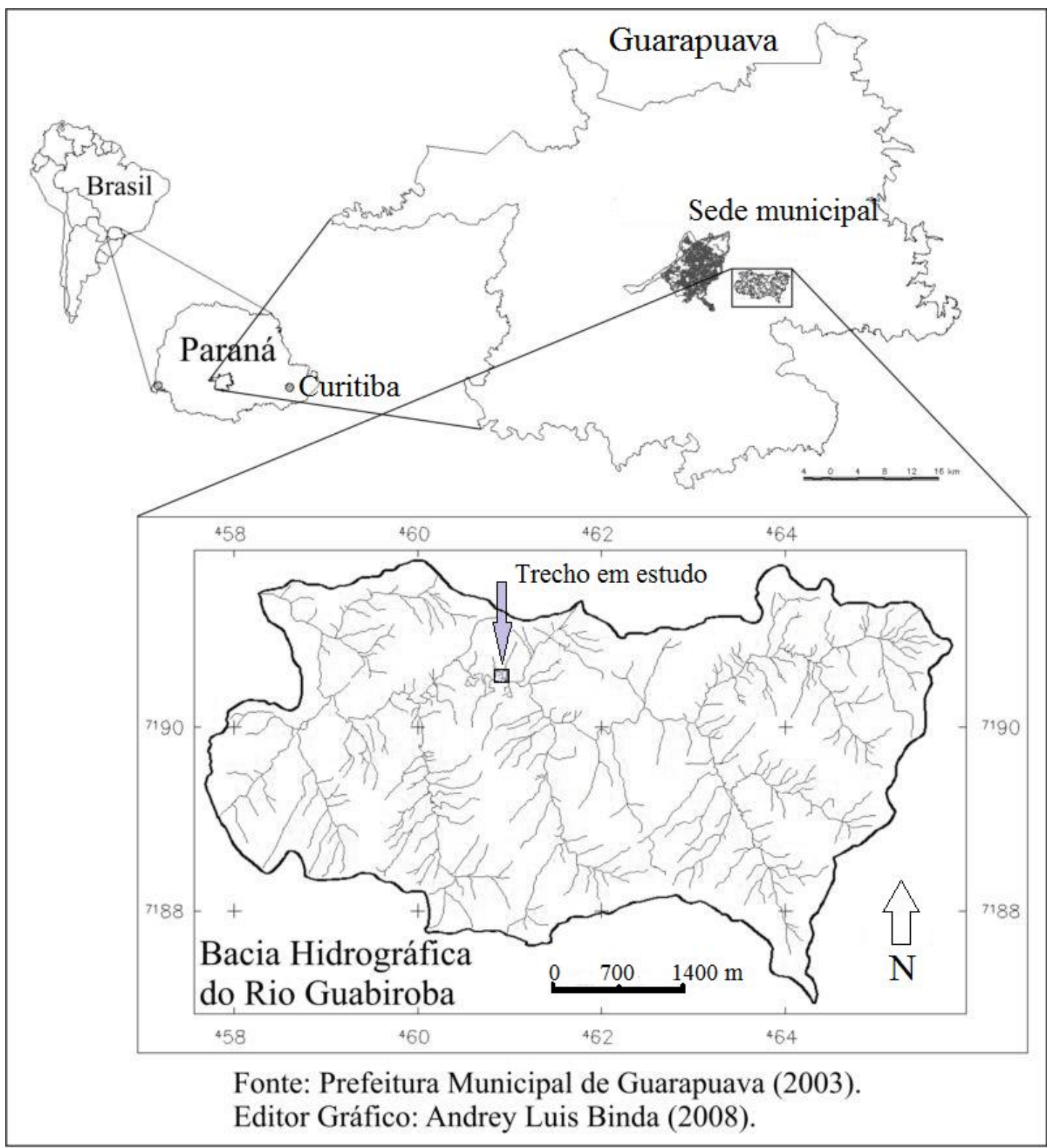

Figura 2: Localização do trecho em estudo na bacia do córrego Guabiroba, município de Guarapuava (PR).

A bacia é esculpida na Unidade Básica Inferior da Formação Serra Geral, caracterizada por rochas de natureza básica-intermediária, normalmente basaltos toleíticos, de granulação muito fina a média, hipocristalinos, de 
coloração cinza escura a negra, maciços ou vesiculares/amigdalóides (NARDY et al., 2002). O clima no município de Guarapuava é subtropical mesotérmico úmido com temperatura média anual de 17으 (THOMAZ e VESTENA, 2003). Quanto a pluviosidade, a média anual é de $1821 \mathrm{~mm}$ (1954-2006) sendo janeiro (192 mm) e outubro (185 mm) os meses mais chuvosos e, abril (129,2 $\mathrm{mm}$ ) e agosto $(99,5 \mathrm{~mm})$ como mais secos (BINDA, 2009).

A vegetação da área de estudo é composta pela associação de campo limpo (Estepe-gramíneo-lenhosa), capões e matas de galeria associadas às florestas de araucárias (Floresta Ombrófila Mista) (MAACK, 1981; BEHLING e PILLAR, 2006).

A classificação alfanumérica de detritos lenhosos proposta por Montgomery (2008) foi aplicada em duas acumulações escolhidas num trecho de 350 m localizado no segmento inferior da bacia do rio Guabiroba (Figuras 2 e 3). Neste trecho, Binda (2009) estudou os efeitos das acumulações de detritos lenhosos sobre os processos erosivos e deposicionais ao longo de um período de 15 meses (Dezembro de 2007 a março de 2009) através de perfis transversais e levantamentos plani-altimétricos. As acumulações dos entulhos arbóreos estão localizadas em segmentos meândricos do rio, onde 0 predomínio da erosão no leito em períodos de cheia gerou habitats aquáticos denominados de depressões ou poços (Figura 4).

O trecho em estudo é classificado segundo o sistema de Rosgen (1994) como do tipo E5, isto é, o canal apresenta baixa declividade, padrão meândrico com seqüência de soleira e depressão, baixa relação largura/profundidade e leito capeado por sedimentos arenosos.

\section{RESULTADOS}

Após a conclusão do monitoramento dos efeitos dos entulhos arbóreos realizado por Binda (2009), os troncos e galhos acumulados nas seções 2 e 3, foram retirados do canal e medidos individualmente o comprimento e a circunferência com auxílio de trena. O diâmetro dos troncos e galhos (D) foi calculado a partir do valor da circunferência $(C)$, aplicando $D=C / \pi$. Antes de 
retirar os detritos lenhosos do seu local original de deposição, foi observada a sua posição visando agrupá-los nas categorias de membros chave, suportado ou solto.

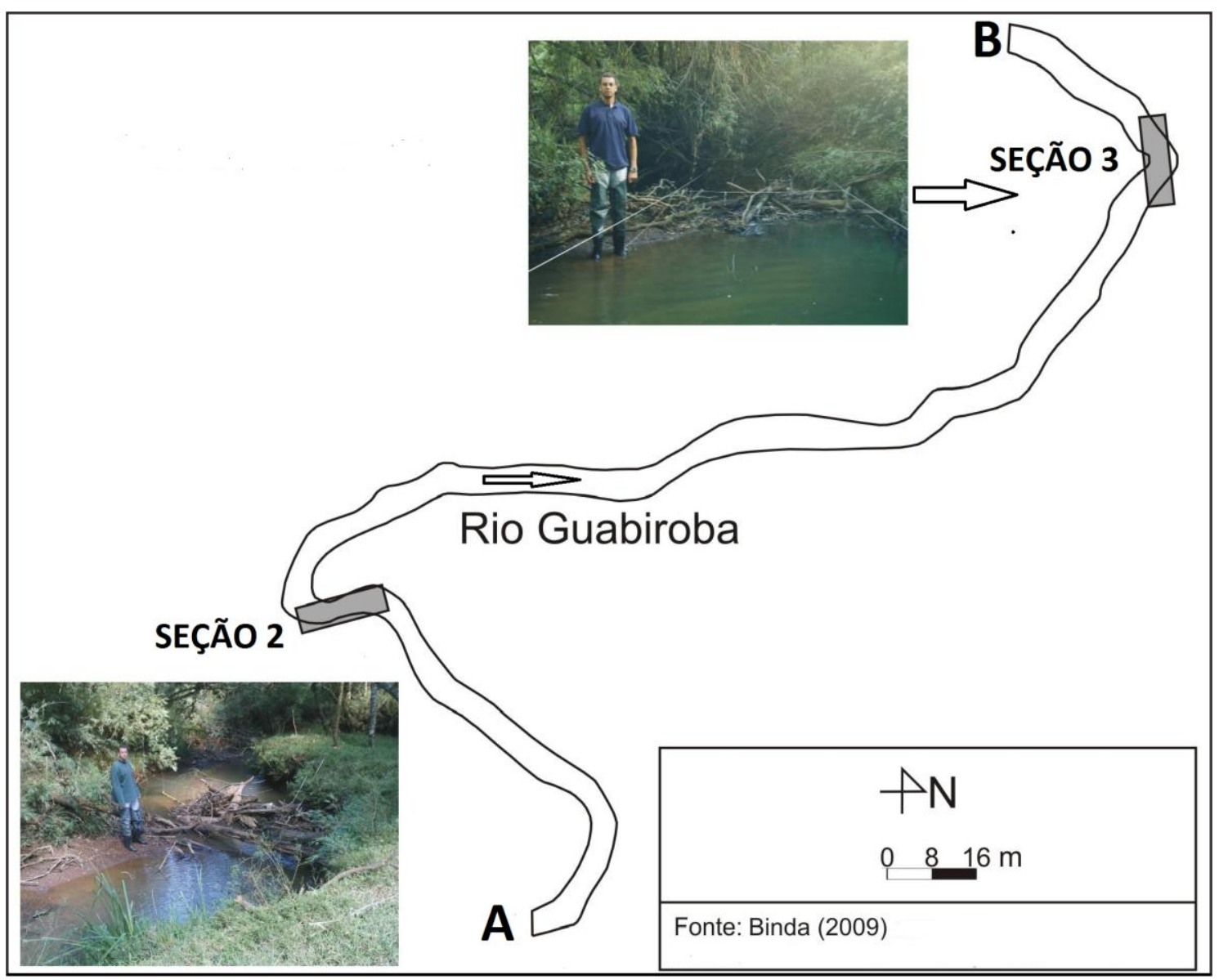

Figura 3: Localização das seções estudadas com entulhos arbóreos no rio Guabiroba, Guarapuava (PR). A localização do trecho é mostrada na figura 2.

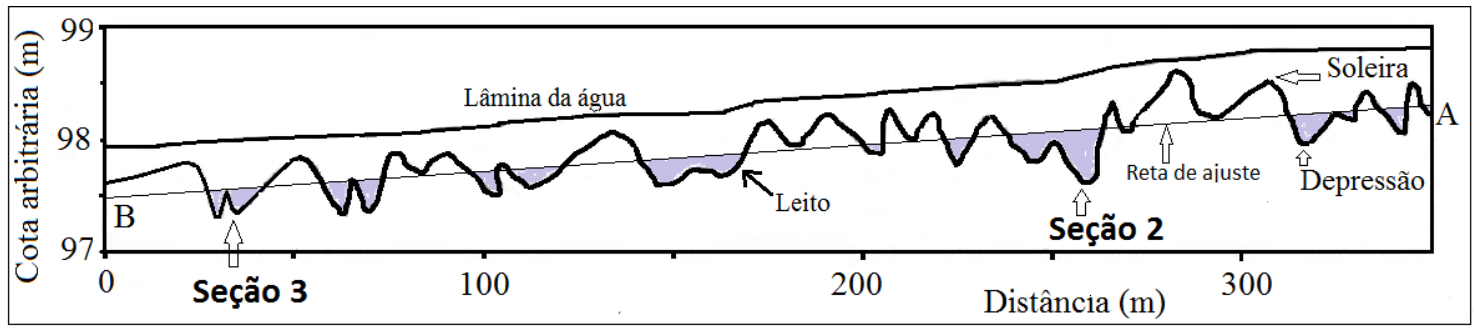

Figura 4: Perfil longitudinal do leito e da lâmina da água no rio Guabiroba entre os pontos A e B na figura 3. (Modificada de Binda e Fernandez, 2010). A reta de ajuste demarca os habitats aquáticos de soleiras ou corredeiras (acima da reta) e depressões ou poços (abaixo). 
Nas figuras 5 e 6 é mostrada a quantidade de detritos lenhosos depositados nas duas seções e sua respectiva classificação nas categorias de membros-chave, suportados e soltos. Nas referidas figuras pode ser observado o predomínio dos troncos dos tipos C1 e C2 entre os membros-chave, ou seja, com comprimento variando de 2 a $4 \mathrm{~m}$ e com diâmetro até de $10 \mathrm{~cm}$ para a classe $\mathrm{C} 1$ e de 10 a $20 \mathrm{~cm}$ para a classe $\mathrm{C} 2$. Entre os membros suportados e soltos dominaram, nas duas seções, os detritos lenhosos da classe B1, ou seja, com comprimento de 1 a $2 \mathrm{~m}$ e com diâmetro de até $10 \mathrm{~cm}$.

O número total de troncos é de 49 unidades na seção 2 e 45 peças na seção 3. A soma dos membros-chave e suportados é maior na seção 3 (33 elementos) em comparação com a seção 2 ( 25 unidades).

Os estudos de Binda (2009) mostraram que no período monitorado (dezembro/2007 a março/2009) predominaram os processos erosivos nas duas seções, intercaladas por esporádicas fases de deposição. As seções estão inseridas em curvas de meandro, onde são formadas depressões em razão do predomínio da erosão nos períodos de chuva (BINDA e FERNANDEZ, 2010). Os levantamentos plani-altimétricos realizados nas seções 2 e 3 (BINDA, 2009; BINDA e FERNANDEZ, 2011) mostraram discrepâncias na magnitude dos processos erosivos no leito em ambas as seções. Na seção 2, a erosão removeu $2,08 \mathrm{~m}^{3}$ de sedimentos, enquanto que na seção 3 a retirada de material alcançou somente $0,96 \mathrm{~m}^{3}$. A baixa magnitude do processo erosivo na seção 3 pode ser atribuída ao maior número de membros-chaves e suportados, servindo de obstáculos na remoção dos sedimentos do leito. 


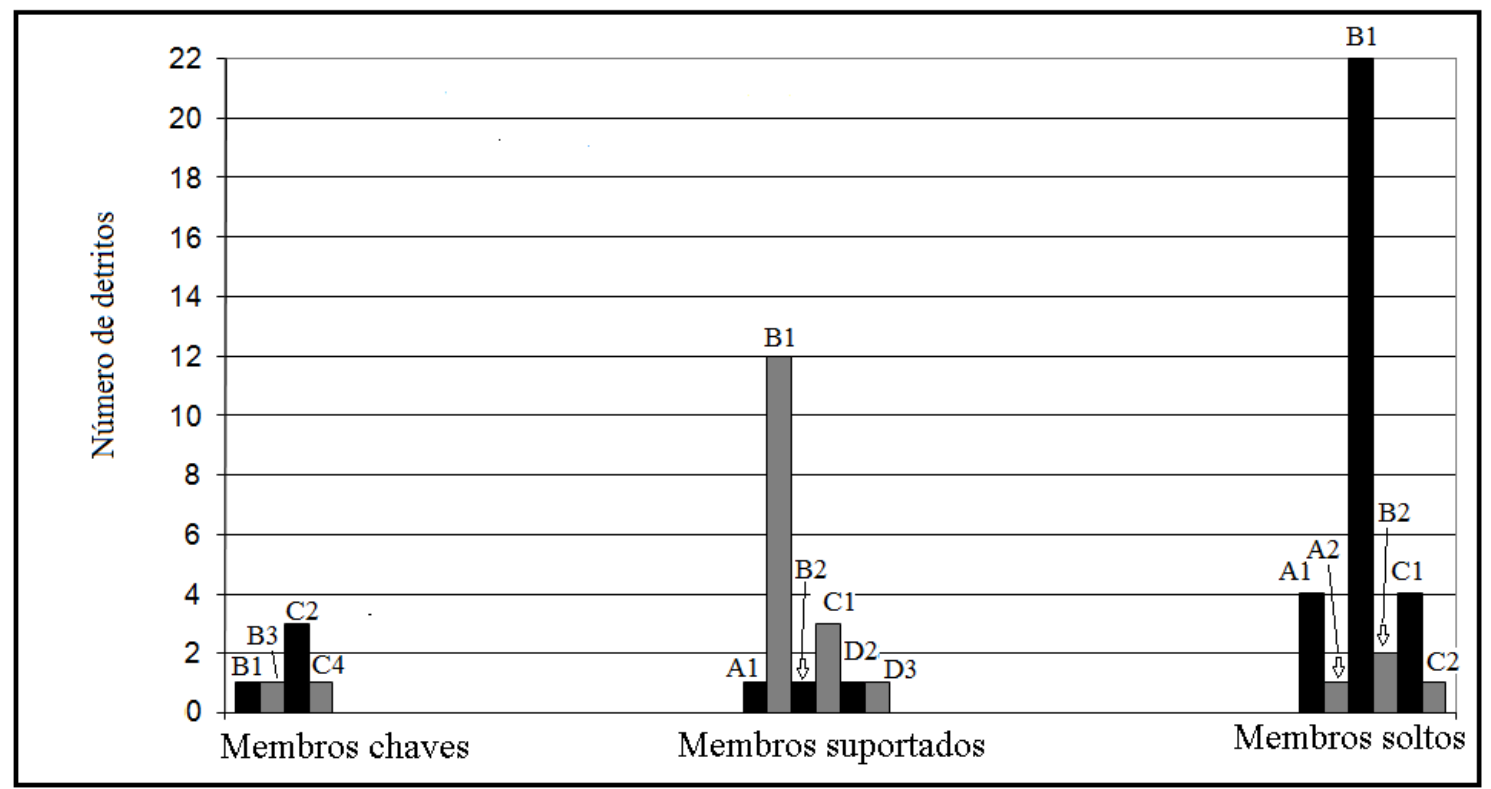

Figura 5: Classificação dos detritos lenhosos acumulados na seção 2 no rio Guabiroba, Guarapuava, PR.

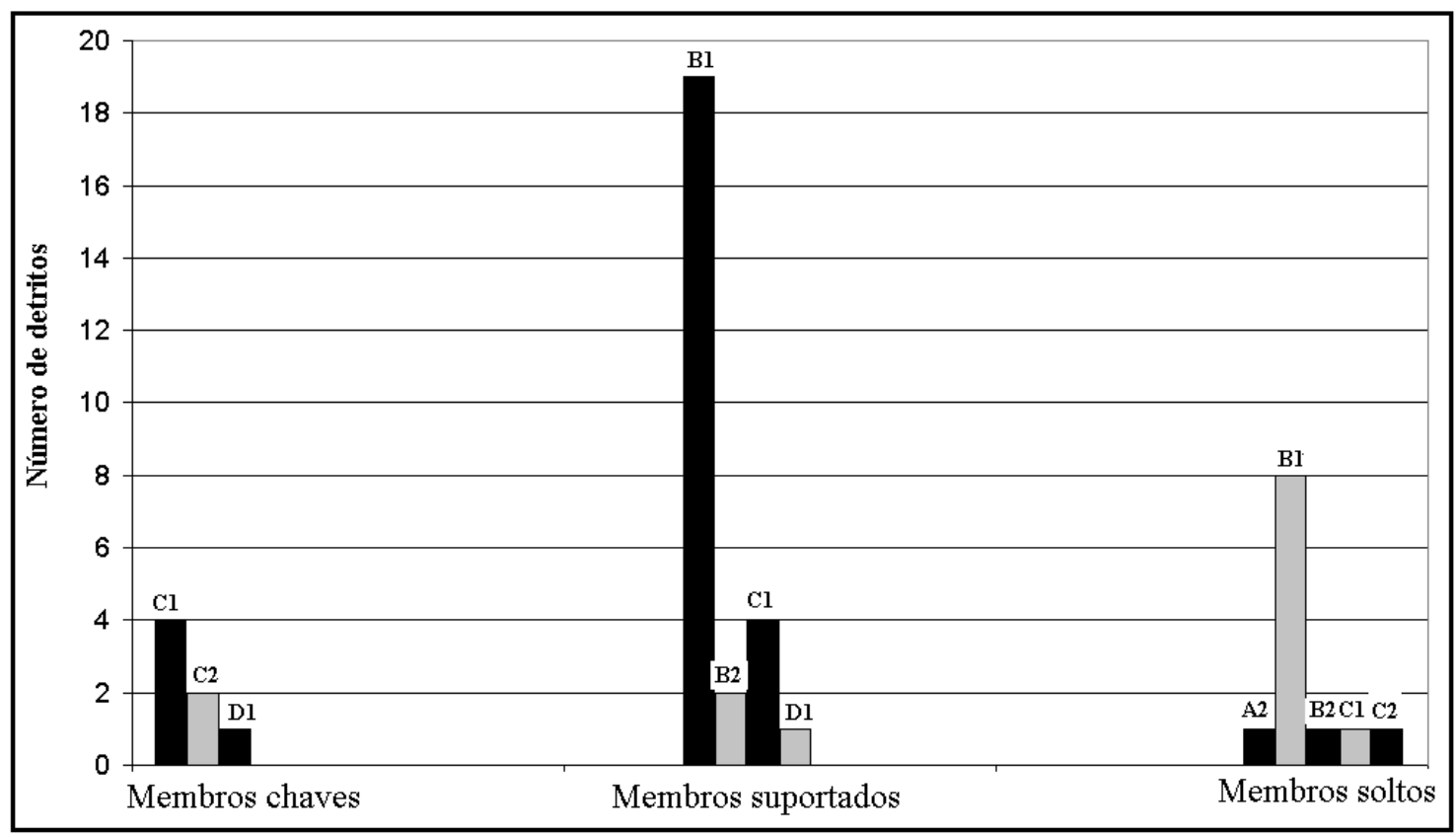

Figura 6: Classificação dos detritos lenhosos acumulados na seção 3 no rio Guabiroba, Guarapuava, PR.

\section{CONSIDERAÇÕES FINAIS}

A aplicação da classificação Montgomery (2008) no rio Guabiroba serviu como exemplo para descrever quantitativamente a população dos resíduos lenhosos amontoados no leito fluvial. As observações sugerem que o volume 
de sedimentos erodidos é menor nas seções com maior quantidade de troncos e galhos fixos no canal (membros-chave e suportados). Esta relação corrobora a importância dos detritos lenhosos na retenção de sedimentos nas bacias hidrográficas.

\section{REFERENCIAS BIBLIOGRÁFICAS}

ABBE, T.B.; MONTGOMERY, D.R. Patterns and processes of wood debris accumulation in the Queets river basin, Washington. Geomorphology, 51: 81107, 2003.

BEHLING, H.; PILLAR, V.D. Late quaternary vegetation, biodiversity and fire dynamics on the southern Brasilian highland and their implication for conservation and management of modern Araucaria forest and grassland ecosystems. Philosophical Transactions of The Royal Society, v. 362, p. 243$251,2006$.

BINDA, A.L. A influência de detritos lenhosos na morfologia e na sedimentologia de leito no Rio Guabiroba, Guarapuava-PR. Dissertação de mestrado. Universidade Estadual do Oeste do Paraná (Unioeste), Campus de Francisco Beltrão. 120 p. 2009.

BINDA, A.L.; FERNANDEZ, O.V.Q. Detritos lenhosos e sequência de soleiras e depressões no rio Guabiroba, Guarapuava-PR. Revista Geografia (Rio Claro), 35 (2): 411-422, 2010.

BINDA, A.L.; FERNANDEZ, O.V.Q. Morfologia de leito e processos erosivodeposicionais em áreas afetadas por acumulações de detritos lenhosos: rio Guabiroba, Guarapuava/PR. Revista Brasileira de Geomorfologia: 12 (2): 105115. 2011.

BINDA, A.L.; LIMA, A.G. Morfologia e processos fluviais: o papel dos detritos lenhosos. Boletim Goiano de Geografia, 28 (2): 59-74, 2008.

BRAGG, D.C.; KERSHNER, J.L.; ROBERTS, D.W. Modeling large woody debris recruitment for small streams of the Central Rocky Mountains. Gen. Tech. Rep. Oregon: USDA, Forest Service, Roocky Mountain Research Station. 2000.

BRAUDRICK, C.A.; GRANT, G.E. Transport and deposition of large woody debris streams: a flume experiment. Geomorphology, 41: 263-283, 2001. 
BROOKS, A.P.; BRIERLEY, G.J.; MILLAR, R.G. The long-term control of vegetation and woody debris on channel and flood-plain evolution: insights from a paired catchment study in southeastern Australia. Geomorphology, 51: 7-29, 2003.

DANIELS, M.D. Distribution and dynamics of large woody debris and organic matter in a low-energy meandering stream. Geomorphology, 77: 286-298, 2006.

DANIELS, M.D.; RHOADS, B.L. Influence of large woody debris obstruction on three-dimensional flow structure in a meander bend. Geomorphology, 51: 159173, 2003.

FARIA, A.P. Influência da vegetação nos processos fluviais de bacias de primeira ordem. Revista Brasileira de Recursos Hídricos, 5 (3): 50-68, 2000.

GIPPEL, C.J., FINLAYSON, B.L., O'NEILL, I. Distribution and hydraulic significance of large woody debris in a lowland Australian river. Hydrobiologia, 318: 179-194, 1994.

GOMI, T.; SIDLE, R.C.; WOODSMITH, R.D.; BRYANT, M.D. Characteristics of channel steps and reach morphology in headwater streams, southeast Alaska. Geomorphology, 51: 225-242, 2003.

GURNELL, A.M., PETTS, G.E., GREGORY, K.J. The role of coarse woody debris in forest aquatic habitats: implications for management. Journal of Aquatic Conservation: Marine and Freshwater Ecosystems 5: 1-24, 1995.

GURNELL, A.M., PIEGAY, H., GREGORY, S.V., SWANSON, F.J. Large wood and fluvial processes. Freshwater Biology, 47: 601-619, 2002.

HASCHENBURGER, J.K.; RICE, S.P. Changes in wood debris and bed material texture in a grave-bed channel. Geomorphology, 60: 241-267, 2004.

KEIM, R.F.; SKAUGSET, A.E.; BATEMAN, D.S. Physical aquatic habitat: II. Pools and cover affected by large woody debris in three western Oregon streams. North American Journal of Fisheries Management, 22: 151-164, 2002.

KELLER, E.A.; SWANSON, F.J. Effects of large organic material on channel form and fluvial processes. Earth Surface Processes, 4: 361-380, 1979.

LENZI, M.A.; COMITI, F.; MAO, L.; ANDREOLI, A.; PECORARI, E.; RIGON, E. El control de detritos leñosos y el manejo de la vegetación en el cauce. Guía Técnica, Dipartimento Territorio e Sistemi Agroforestali; Università degli Studi di Padova. 185 p., 2006.

LESTER, R.; WRIGHT, W.; LENNON, M.J. Determining target loads of large and small wood for stream rehabilitation in high-rainfall agricultural regions of Victoria, Australia. Ecological Engineering, 28: 71-78, 2006. 
LINSTEAD, C.; GURNELL, A.M. Large woody debris in British headwater rivers: physical habitat role and management guidelines. Birmingham: University of Birmingham/School of Geography \& Environmental Sciences/Environment Agency, 36 p. 1999.

MAACK, R. Geografia física do Estado do Paraná. 2a ed., Rio de Janeiro: J. Olympio; Curitiba: Secretaria da Cultura e do Esporte do Governo do Estado do Paraná. 1981.

Mac NALLY, R., PARKINSON, A., HORROCKS, G., CONOLE, L., TZAROS, C. Relationships between terrestrial vertebrate diversity, abundance and availability of coarse woody debris on southeastern Australian floodplains. Biological Conservation 99: 191-205, 2001.

MONTGOMERY, D.R. A simple alphanumeric classification of wood debris size and shape. Stream Notes, Stream Systems Technological Center. Disponível em: http://www.stream.fs.fed.us. Acesso em: 2 de dezembro de 2008. 2008.

MONTGOMERY, D.R.; COLLINS, B.D.; BUFFINGTON, J.M.; ABBE, T.B. Geomorphic effects of wood in rivers. American Fisheries Symposium, p. 1-27, 2003.

MONTGOMERY, D.R.; PIEGAY, H. Wood in rivers: interactions with channel morphology and processes. Geomorphology 51, 1-5, 2003.

NARDY, A.J.R.; OLIVEIRA, M.A.F.; BETANCOURT, R.H.S.; VERDUGO, D.R.H.; MACHADO, F.B. Geologia e Estratigrafia da Formação Serra Geral. Geociências (Unesp, São Paulo): 21: 15-32, 2002.

PETTIJHON, F.J.; POTTER, P.E.; SIEVER, R. Sand and sandstone. SpringerVerlag, New York, 618 p., 1973.

ROSGEN, D.L. A classification of natural rivers. Catena, 21: 169-199, 1994.

SUNDBAUM, K.; NAESLUND, I. Effects of woody debris on the growth and behaviour of brown trout in experimental stream channels. Canadian Journal of Zoology 76: 56-61, 1998.

THOMAZ, E.L.; VESTENA, L.R. Aspectos climáticos de Guarapuava-PR. Guarapuava: Editora Unicentro, 2003. 106 p.

WALLERSTEIN, N.P.; THORNE, C.R. Influence of large woody debris on morphological evolution of incised, sand-bed channels. Geomorphology, 57: 5373, 2004.

WEBB, A.A.; ERSKINE, W.D. Distribution, recruitment, and geomorphic significance of large woody debris in an alluvial forest stream: Tonghi Creek, southeastern Australia. Geomorphology, 51: 109-126, 2003. 
WOHL, E.E.; CENDERELLI, D.A.; DWIRE, K.A.; RYAN-BURKETT, S.E.; YOUNG, M.K.; FAUSCH, K.D. Conducting large in-stream wood studies: a call for common metrics. Stream Notes, Stream Systems Technological Center. Disponível em: http://www.stream.fs.fed.us. Acesso em: 2 de setembro de 2010, 2010.

YOUNG, M.K.; MACE, E.A.; ZIEGLER, E.T.; SUTHERLAND, E.K. Characterizing and contrasting instream and riparian coarse wood in western Montana basins. Forest Ecology and Management, 226: 26-40, 2006. 